PROCEEDINGS OF THE

AMERICAN MATHEMATICAL SOCIETY

Volume 129, Number 4 , Pages 1105-1112

S 0002-9939(00)05757-9

Article electronically published on October 11, 2000

\title{
GLOBAL LOWER BOUND FOR THE HEAT KERNEL OF $-\Delta+\frac{c}{|x|^{2}}$
}

\author{
QI S. ZHANG
}

(Communicated by David S. Tartakoff)

\begin{abstract}
We obtain global in time and qualitatively sharp lower bounds for the heat kernel of the singular Schrödinger operator $-\Delta+\frac{a}{|x|^{2}}$ with $a>0$. Here $\Delta$ is either the Laplace-Beltrami operator or the Laplacian on the Heisenberg group. This complements a recent paper by P. D. Milman and Yu. A. Semenov in which an upper bound was found. The above potential is interesting because it is a border line case where both the strong maximum principle and Gaussian bounds fail.
\end{abstract}

\section{InTRODUCTION}

We obtain global in time and qualitatively sharp lower bounds for the fundamental solution of the singular parabolic equation including $\Delta u-V u-\partial_{t} u=0$. The potentials $V=V(x, t)$ have singularities of the type $\frac{a}{|x|^{2}}$ with $a>0$. It is well known that these potentials belong to a borderline case where both the strong maximum principle and Gaussian bounds in [A] or [LY] fail. Such $V$ also lie outside the Kato class potentials which have been studied extensively. The Cauchy problem with similar potentials was studied by Baras and Goldstein [BG]. In [DS], Davies and Simon obtained bounds for the fundamental solutions when $a<0$ and $V$ is a bounded function decaying like $a /|x|^{2}$ near infinity.

In an interesting recent paper $\mathrm{MS}$, P. D. Milman and Yu. A. Semenov studied the fundamental solutions of the above equation in the Euclidean case. They obtained lower and upper bounds for the fundamental solution when $-c_{0}<a<0$ and upper bounds when $0<a<c_{0}$ where $c_{0}$ is a dimensional constant. More precisely they have, among other things,

Theorem (P. Milman and $\mathrm{Yu}$ A. Semenov). Let $\mathbf{M}=\mathbf{R}^{n}$ with $n \geq 3$. Suppose there are constants $\lambda_{i}, \gamma, \beta ; 0 \leq \gamma \leq \beta<1$, such that $\lambda_{1}+\gamma((n-2) / 2)^{2}|x|^{-2} \leq$ $V(x) \leq \lambda_{2}+\beta((n-2) / 2)^{2}|x|^{-2}$. There are constants $c_{i}=c_{i}(\epsilon, q)>0$ and $\omega_{i}=\omega_{i}(\epsilon)$ such that the inequalities hold for all $x, y$ and $t>0$,

$$
\begin{aligned}
& e^{-t(-\Delta-V)}(x, y) \leq c_{1} t^{-n / 2} \exp \left[\omega_{1} t-c_{2} \frac{|x-y|^{2}}{4 t}\right] \phi_{2, \epsilon}(x) \phi_{2, \epsilon}(y), \\
& e^{-t(-\Delta+V)}(x, y) \leq c_{3} t^{-n(1+q) /(2 q)} \exp \left[\omega_{2} t-c_{4} \frac{|x-y|^{2}}{4 t}\right] \phi_{3, \epsilon}(x) \phi_{3, \epsilon}(y) .
\end{aligned}
$$

Received by the editors June 30, 1999.

1991 Mathematics Subject Classification. Primary 35K10, 35K65. 
Here $\epsilon \in\left(0, \min \left\{1,\left(\frac{1}{\beta}-1\right)\right\}\right)$,

$$
\phi_{i, \epsilon}=|x|^{-\sigma_{i}(\epsilon)} \text { if }|x| \leq 1 \text { and } \phi_{i, \epsilon}=1 \text { if }|x| \geq 1, i=2,3 \text {, }
$$

where $\sigma_{3}=-\sigma_{2}$ and $\sigma_{2}=\frac{n-2}{2(1+\epsilon)}(1-\sqrt{1-\beta-\epsilon \beta})$, and $q \in\left(2, n / \sigma_{1}\right), \sigma_{1}=\frac{n-2}{2(1+\epsilon)} \times$ $(-1+\sqrt{1+\gamma-\epsilon \gamma})$.

By the upper bound for $e^{-t(-\Delta+V)}$ and the Trotter product (or the FeynmanKac) formula, one can immediately obtain a lower bound for $e^{-t(-\Delta-V)}$. However the lower bound for $e^{-t(-\Delta+V)}$ was left open in [MS]. The first goal of the paper is to prove a lower bound complementing the upper bound in $\mathrm{MS}$. Next we also obtain a lower bound that is global in time. Finally we show that the lower bound actually holds in a more general setting of degenerate parabolic equations or equations on some manifolds.

In this paragraph we lay out a number of basic assumptions and notations to be used throughout the paper. We are mainly concerned with the fundamental solutions $G=G(x, t ; y, 0)$ to the following equation:

$$
\Delta u-V u-\partial_{t} u=0 \text { in } \mathbf{M} \times(0, \infty) .
$$

Here $\mathbf{M}$ is an $n(\geq 3)$ dimensional complete noncompact manifold with nonnegative Ricci curvature. Even though the curvature condition can be relaxed further we will not seek the full generality in this paper. Note that $G=e^{-t(-\Delta+V)}$ if $V$ is independent of time.

From now on 0 will be a reference point on $\mathbf{M}$ and $d(x, y), d(x)$ will be the distance between $x, y$ and between $x, 0$, respectively. The notation $Q_{r}(x, t)$ will be used to denote the parabolic cube $B(x, r) \times\left(t-r^{2}, t\right)$. We will use $c, C, c_{1}, C_{1}, \ldots$, to denote generic positive constants.

The potential $V=V(x, t)$ has singularities of the type $\frac{a}{d(x)^{2}}$ with $a>0$.

We also need the following

Assumption 1.1. For any $r>0, \partial B(0, r)$ can be covered by a chain of geodesic balls satisfying (i) each ball in the chain is of radius $r / 4$; (ii) the union of the balls is a connected set; (iii) the distance from the chain to 0 is not smaller than $r / 4$; (iv) the number of balls in the chain is independent of $r$.

When $\mathbf{M}=\mathbf{R}^{n}$ the above assumptions clearly hold. However $S^{2} \times \mathbf{R}$ with the standard metric does not satisfy (ii).

The following theorem is the main result of the paper.

Theorem 1.1 (the lower bound). Under assumption (1.1), suppose, for $a>0$, $V(x, t) \leq \frac{a}{d^{2}(x)}$ and $G \geq 0$. Then there exist positive constants $c_{1}, c_{2}$ such that, for all $x, y$ and $t>0$,

$$
G(x, t ; y, 0) \geq c_{1} \frac{w\left(x, t^{1 / 2}\right)}{\sqrt{\left|B\left(x, t^{1 / 2}\right)\right|}} \frac{w\left(y, t^{1 / 2}\right)}{\sqrt{\left|B\left(y, t^{1 / 2}\right)\right|}} e^{-c_{2} \frac{d(x, y)^{2}}{t}} .
$$

Here $w\left(x, t^{1 / 2}\right)=\min \left\{\left(\frac{d(x)}{t^{1 / 2}}\right)^{\alpha}, 1\right\}$ where $\alpha=c_{3}+c_{4} a$ and $c_{3}, c_{4}$ depend only on M.

Remark 1.1. One can notice that the upper bound in [MS] and the lower bound in this paper have slightly different weight functions. It seems that the term $d(x) / t^{1 / 2}$ in the lower bound is necessary because the equation $\Delta u-\frac{a}{|x|^{2}} u-u_{t}=0$ is invariant under parabolic dilation $x \rightarrow c x, t \rightarrow c^{2} t$, in $\mathbf{R}^{n} \times(0, \infty)$. 
Combining the lower bound in the above theorem with the upper bound in MS, we now know that the heat kernel $G$ of $-\Delta+V(x)$ is the multiple of a standard Gaussian with a weight function of $t, d(x)$ and $d(y)$ and having polynomial decay in time. At this moment the constants $c_{3}, c_{4}$ in the lower bound cannot be explicitly computed as for the upper bound in the Euclidean case. However in the the general context of heat equations on manifolds, it seems impossible to have a precise estimate anyway. An advantage of the current lower bound is that it is globally sharp since it does not contain the term $e^{-w t}$. When $V$ is in the Kato class such a global bound was first observed in $[\mathrm{Se}]$. Moreover, in contrast to the case of upper bound, no assumptions on the size of $a$ is needed for the lower bound.

Remark 1.2. Let us recall a simple inequality which affords us to switch from $x$ to $y$ freely in a Gaussian when necessary:

$$
\frac{1}{\left|B\left(x, t^{1 / 2}\right)\right|} e^{-c d(x, y)^{2} / t} \leq \frac{C_{\epsilon}}{\left|B\left(y, t^{1 / 2}\right)\right|} e^{-(c-\epsilon) d(x, y)^{2} / t},
$$

where $0<\epsilon<c$. The proof is omitted.

Remark 1.3. The result of the paper covers an important class of degenerate equations. As one can see in section 2, the proof of the lower bound depends only on the doubling property of the metric balls and the standard parabolic Harnack inequality and Theorem 1.1. Therefore the lower bound also holds when $\mathbf{M}$ is replaced by a Lie group of polynomial growth and $\Delta$ is replaced by Hörmander's sum of square operators of left invariant vector fields (see [S-C2] $)$. In particular, Theorem 1.1 holds for the heat equation on the Heisenberg group.

We mention that the idea in proving the lower bound is to use a carefully constructed Harnack chain argument which can capture the information on the potential.

\section{Proof of Theorem 1.1}

The proof of the theorem is preceded by three lemmas which utilize a carefully designed Harnack chain argument to prove the lower bound for $G=G(x, t ; y, 0)$ in different portions of $\mathbf{M} \times(0, \infty) \times \mathbf{M}$. The portions are considered in the following order. Lemma 2.1 covers $x=y, d(x) \geq a_{1} t^{1 / 2}$; Lemma 2.2 covers $d(x)^{2} \leq a_{1} t$ and $d(y)^{2} \leq a_{2} t$; Lemma 2.3 is for $d(x)^{2} \geq a_{1} t$ and $d(y)^{2} \geq a_{2} t$; here $a_{1}$ and $a_{2}$ are positive constants. Some overlaps among the lemmas will help explain the procedure. After proving the lemmas we will be ready to prove the theorem.

Throughout the section we assume that $V(x, t) \leq \frac{a}{d(x)^{2}}$ with $a>0$.

Lemma 2.1. Suppose that $d(x)^{2} \geq a_{1}$ t for $a_{1}>0$. Then

$$
G(x, t ; x, 0) \geq \frac{C}{\left|B\left(x, t^{1 / 2}\right)\right|} .
$$

Proof. Without loss of generality we take $a_{1}=1$. We pick a point $x_{1}$ such that $d\left(x_{1}\right) \geq t$.

Let $\phi \in C_{0}^{\infty}\left(B\left(x_{1}, t^{1 / 2} / 2\right)\right)$ be such that $\phi(x)=1$ when $x \in B\left(x_{1}, t^{1 / 2} / 4\right)$ and $0 \leq \phi \leq 1$ everywhere. Consider the function

$$
u(x, t)=\int_{\mathbf{M}} G(x, t ; y, 0) \phi(y) d y .
$$


As in S-C1, we extend $u$ by assigning $u(x, t)=1$ when $t<0$ and $x \in B\left(x_{1}, t^{1 / 2} / 4\right)$; then $u$ is a positive solution of $(1)$ in $B\left(x_{1}, t^{1 / 2} / 4\right) \times(-\infty, \infty)$. Here we take $V(x, t)=0$ when $t<0$ and we note that no continuity of $V$ is needed. For any $y \in B\left(x_{1}, t^{1 / 2} / 2\right)$, we have

$$
d(y, 0) \geq d\left(0, x_{1}\right)-d\left(x_{1}, y\right) \geq t-t^{1 / 2} / 2 \geq t / 2 .
$$

Hence, by the decay condition on $V$, there is a constant $C>0$ such that

$$
m \equiv \sup _{B\left(x, t^{1 / 2} / 2\right)}|V(y, s)| \leq C / t
$$

Using twice the Harnack inequality as stated, for example, in Theorem 5.3 in [S-C1], we obtain

$$
\begin{gathered}
u\left(x_{1}, 0\right) \leq C e^{m t} u\left(x_{1}, t / 4\right) \leq C u\left(x_{1}, t / 4\right), \\
G\left(y, t / 4 ; x_{1}, 0\right) \leq C e^{m t} G\left(x_{1}, t ; x_{1}, 0\right) \leq C G\left(x_{1}, t ; x_{1}, 0\right),
\end{gathered}
$$

for $y \in B\left(x_{1}, t^{1 / 2} / 2\right)$. Hence

$$
\begin{aligned}
1 & =u\left(x_{1}, 0\right) \leq C u\left(x_{1}, t / 4\right)=C \int_{B\left(x_{1}, t^{1 / 2} / 2\right)} G\left(x_{1}, t / 4 ; y, 0\right) \phi(y) d y \\
& =C \int_{B\left(x_{1}, t^{1 / 2} / 2\right)} G\left(y, t / 4 ; x_{1}, 0\right) \phi(y) d y \leq C G\left(x_{1}, t ; x_{1}, 0\right) \int_{B\left(x_{1}, t^{1 / 2} / 2\right)} \phi(y) d y \\
& \leq C G\left(x_{1}, t ; x_{1}, 0\right)\left|B\left(x_{1}, t^{1 / 2}\right)\right| .
\end{aligned}
$$

Since $x_{1}$ is arbitrary, the lemma is proven.

Lemma 2.2. Suppose $d(x)^{2}, d(y)^{2} \leq a_{1} t$. Then there exist positive constants $c_{1}$, $\alpha$ such that

$$
G(x, t ; y, 0) \geq c_{1}\left(\frac{t^{1 / 2}}{d(x)}\right)^{-\alpha}\left(\frac{t^{1 / 2}}{d(y)}\right)^{-\alpha} \frac{1}{\left|B\left(x, t^{1 / 2}\right)\right|}
$$

where $\alpha$ is a linear function of the constant a in Theorem 1.1.

Proof. It suffices to prove the lemma when $a_{1}=1$. Let $\gamma$ be a minimal geodesic connecting 0 and $x$. We extend $\gamma$ to infinity and pick $z$ on $\gamma$ such that $d(x, z)=t^{1 / 2}$ and $d(0, z)=d(0, x)+d(x, z)$. For simplicity we parameterize $\gamma$ by length and take $\gamma(0)=x$ and $\gamma\left(t^{1 / 2}\right)=z$.

For nonnegative integers $i$ we write $y_{i}=\gamma\left(2^{i} \delta d(x)\right)$ with $\delta \in[1 / 2,1]$ to be determined later. Clearly $y_{i}, y_{i+1} \in \bar{B}\left(y_{i+1}, 2^{i} \delta d(x)\right) \subset B\left(y_{i+1}, 2^{i} \delta d(x) 11 / 10\right)$. For $y^{\prime} \in B\left(y_{i+1}, 2^{i} \delta d(x) 11 / 10\right)$, we have

$$
d\left(y^{\prime}, 0\right) \geq d\left(0, y_{i+1}\right)-d\left(y_{i+1}, y^{\prime}\right) \geq\left(2^{i+1}-2^{i} 11 / 10\right) \delta d(x)=2^{i} \delta d(x) 9 / 10 .
$$

Therefore, there is $C>0$ such that

$$
\beta_{i} \equiv \sup _{B\left(y_{i+1}, 2^{i} \delta d(x) 11 / 10\right) \times(0, \infty)}\left|V\left(y^{\prime}, t\right)\right| \leq \frac{C a}{2^{2 i} \delta^{2} d(x)^{2}} .
$$

Suppose $u$ is a positive solution to (1.1). By the Harnack inequality stated in Corollary 5.4 of [S-C1], we have for $x^{\prime}, y^{\prime} \in \bar{B}\left(y_{i+1}, 2^{i} \delta d(x)\right)$ and $s>s^{\prime}$,

$$
\ln \left[u\left(y^{\prime}, s^{\prime}\right) / u\left(x^{\prime}, s\right)\right] \leq C\left[\frac{d^{2}\left(x^{\prime}, y^{\prime}\right)}{s-s^{\prime}}+\left(\beta_{i}+\frac{1}{s^{\prime}}\right)\left(s-s^{\prime}\right)\right] .
$$


Taking $y^{\prime}=y_{i+1}, x^{\prime}=y_{i}, s^{\prime}=2 t-2^{2(i+1)} \delta^{2} d(x)^{2}$ and $s=2 t-2^{2 i} \delta^{2} d(x)^{2}$, we have, for a

$$
\begin{gathered}
C_{2}=c_{3} e^{c_{4} a}>0, \\
u\left(y_{i+1}, 2 t-2^{2(i+1)} \delta^{2} d(x)^{2}\right) \leq C_{2} u\left(y_{i}, 2 t-2^{2 i} \delta^{2} d(x)^{2}\right) .
\end{gathered}
$$

Since $t^{1 / 2} / d(x) \geq 1$, there exists a $\delta \in[1 / 2,1]$ such that

$$
k=\log _{2} \frac{t^{1 / 2}}{\delta d(x)}
$$

is an integer. For such an integer $k$ we have

$$
y_{k}=\gamma\left(2^{k} \delta d(x)\right)=\gamma\left(t^{1 / 2}\right)=z, 2 t-2^{2 k} \delta^{2} d(x)^{2}=t .
$$

Iterating (2.1) $k$ times we obtain

$$
u(z, t) \leq C_{2}^{c \log _{2}\left(t^{1 / 2} /(\delta d(x))\right)}=\left[c_{3} e^{c_{4} a}\right]^{c \log _{2}\left(t^{1 / 2} /(\delta d(x))\right)} u(x, 2 t) .
$$

Therefore there exists $\alpha=c_{5}+c_{6} a>0$ such that

$$
C\left(t^{1 / 2} / d(x)\right)^{-\alpha} u(z, t / 2) \leq u(x, t) .
$$

In the above $\delta \in[1 / 2,1]$ is absorbed into $C$. Repeating the above process, we have, for a $z_{1}$ such that $d\left(y, z_{1}\right)=t^{1 / 2}$ and $d(z, 0)=d(y, 0)+d\left(y, z_{1}\right)$,

$$
C\left(t^{1 / 2} / d(x)\right)^{-\alpha} u\left(z_{1}, t / 4\right) \leq u(y, t / 2) .
$$

Applying the above inequalities to the first entries of $G$, we have

$$
\begin{aligned}
G(x, t ; y, 0) & \geq C\left(t^{1 / 2} / d(x)\right)^{-\alpha} G(z, t / 2 ; y, 0) \\
& =C\left(t^{1 / 2} / d(x)\right)^{-\alpha} G(y, t / 2 ; z, 0) \\
& \geq C\left(t^{1 / 2} / d(x)\right)^{-\alpha}\left(t^{1 / 2} / d(y)\right)^{-\alpha} G\left(z_{1}, t / 4 ; z, 0\right) .
\end{aligned}
$$

Next we need to find a lower bound for $G\left(z_{1}, t / 4 ; z, 0\right)$. From construction, we have $t^{1 / 2} \leq d(z) \leq 2 t^{1 / 2}$ and $t^{1 / 2} \leq d\left(z_{1}\right) \leq 2 t^{1 / 2}$. By Theorem 1.1 we can form a chain of fixed number of parabolic cubes satisfying:

(i) each cube is of size $t^{1 / 2} / 4$ in the spatial direction and $t / 16$ in the time direction;

(ii) the first cube covers $\left(z_{1}, t / 4\right)$ and the last covers $(z, t / 8)$;

(iii) adjacent cubes have a gap of $c t$ in the time direction and the centers of the adjacent cubes have a distance no greater than $c t^{1 / 2}$ in the spatial direction;

(iv) for each $(z, \tau)$ in the cubes $d(z)^{2} \geq c t^{1 / 2}$ for $c>0$.

Along this chain, we have, by (iv),

$$
V(z, \tau) \leq \frac{a}{d(z)^{2}} \leq \frac{c}{t}
$$

By the Harnack inequality, for any $\left(z_{i}, \tau_{i}\right)$ in the $i-t h$ cube we have

$$
G\left(z_{i+1}, \tau_{i+1} ; z, 0\right) \leq e^{C \sup V t} G\left(z_{i}, \tau_{i} ; z, 0\right) .
$$

Since $V t \leq C$ along the chain, we have

$$
G\left(z_{i+1}, \tau_{i+1} ; y, 0\right) \leq C G\left(z_{i}, \tau_{i} ; y, 0\right) .
$$

Multiplying the above together we have

$$
G(z, t / 8 ; z, 0) \leq C G\left(z_{1}, t / 4 ; z, 0\right)
$$

since there are fixed number of cubes. 
Since $t^{1 / 2}=d(x, z) \leq d(z, 0) \leq d(z, x)+d(x, 0) \leq 2 t^{1 / 2}$, by Lemma 2.1 and the doubling property

$$
G(z, t / 8 ; z, 0) \geq \frac{C}{|B(z, t)|} \geq \frac{C}{|B(x, t)|} .
$$

Combining (2.5)-(2.8) we have

$$
G(x, t ; y, 0) \geq\left(t^{1 / 2} / d(x)\right)^{-\alpha}\left(t^{1 / 2} / d(y)\right)^{-\alpha} \frac{C}{|B(x, t)|} .
$$

By (2.3) $\alpha$ is a linear function of $a$.

Lemma 2.3. Suppose $d(x, y)^{2} \geq a_{1} t, d(x)^{2} \geq a_{2} t$ and $d(y)^{2} \geq a_{2} t$. Then there exist positive constants $c_{1}, c_{2}$ such that

$$
G(x, t ; y, 0) \geq c_{1} \frac{e^{-c_{2} d(x, y)^{2} / t}}{\left|B\left(x, t^{1 / 2}\right)\right|} .
$$

Proof. Without loss of generality we take $a_{1}=a_{2}=1$. Since $d(x, y)^{2} \geq t$, we can form a chain of parabolic cubes such that

(i) each cube is of size $\delta t / d(x, y)$ in the spatial direction and $[\delta t / d(x, y)]^{2}$ in the time direction;

(ii) the first cube covers $(x, t)$ and the last covers $(y, t / 2)$;

(iii) adjacent cubes have a gap of $c[\delta t / d(x, y)]^{2}$ in the time direction and the centers of the adjacent cubes have a distance no greater than $c \delta t / d(x, y)$ in the spatial direction;

(iv) for each $(z, \tau)$ in the cubes $d(z)^{2} \geq c t$ for $c>0$ depending on $b$ and $\delta$;

(v) the number of cubes along this chain is chosen as $k=c d(x, y)^{2} / t$. In the above $\delta>0$ is a fixed number.

Let us provide the detail of the construction. Since $d(x)^{2}, d(y)^{2} \geq t$, by Theorem 1.1 we can find a curve $l$ in $\mathbf{M}$ connecting $x$ and $y$ such that $L(l) \leq A_{0} d(x, y)$ and for each point $z$ on the curve $d(z)^{2}=d(z, 0)^{2} \geq A_{1} t$. Here $A_{0}$ and $A_{1}$ are positive constants. Choosing the center of the parabolic cubes along $l \times[t / 2, t]$ one can easily construct a chain of parabolic cubes satisfying (i), (ii), (iii) and (v). That (iv) also holds follows from the following argument.

Let $(z, s)$ be a point in one of the cubes. Then there exists a $z_{0} \in l$ such that $d\left(z, z_{0}\right) \leq \delta t / d(x, y)$. Hence $d\left(z, z_{0}\right)^{2} \leq \delta^{2} t^{2} / d(x, y)^{2} \leq \delta^{2} t$. Therefore

$$
d(z, 0)^{2} \geq C\left[d\left(z_{0}, 0\right)^{2}-d\left(z, z_{0}\right)^{2}\right] \geq C\left[A_{1} t-\delta^{2} t\right] \geq c t
$$

when $\delta$ is a small fixed number.

Along this chain, we have, by (iv),

$$
V(z, \tau) \leq \frac{a}{d(z)^{2}} \leq \frac{c}{t}
$$

By the Harnack inequality, for any $\left(z_{i}, \tau_{i}\right)$ in the $i$-th cube we have

$$
G\left(z_{i+1}, \tau_{i+1} ; y, 0\right) \leq e^{C \sup V[t / d(x, y)]^{2}} G\left(z_{i}, \tau_{i} ; y, 0\right) .
$$

Since $V[t / d(x, y)]^{2} \leq \frac{a}{t}[t / d(x, y)]^{2}=\frac{a t}{d(x, y)^{2}} \leq C$ along the chain, we have

$$
G\left(z_{i+1}, \tau_{i+1} ; y, 0\right) \leq C G\left(z_{i}, \tau_{i} ; y, 0\right) .
$$

Multiplying the above together we have, by (v),

$$
G(y, t / 2 ; y, 0) \leq C^{c d(x, y)^{2} / t} G(x, t ; y, 0) .
$$


Since $d(y)^{2} \geq t$ we have, by Lemma 2.1,

$$
G(y, t / 2 ; y, 0) \geq \frac{C}{\left|B\left(y, t^{1 / 2}\right)\right|} .
$$

Combining the last two inequalities we have

$$
G(x, t ; y, 0) \geq \frac{C}{\left|B\left(y, t^{1 / 2}\right)\right|} e^{-c d(x, y)^{2} / t} .
$$

By Remark 1.2 this proves the lemma.

Now we are ready to give a

Proof of Theorem 1.1. Part I. When $d(x, y)^{2} \leq t$.

By Remark 1.2 we only need to show that:

Suppose $d(x, y)^{2} \leq t$. Then there exist positive constants $c_{1}, c_{2}$ such that

$$
G(x, t ; y, 0) \geq \min \left\{\left(\frac{t^{1 / 2}}{d(x)}\right)^{-c_{2}}, 1\right\} \min \left\{\left(\frac{t^{1 / 2}}{d(y)}\right)^{-c_{2}}, 1\right\} \frac{c_{1}}{\left|B\left(x, t^{1 / 2}\right)\right|} .
$$

If both $d(x)^{2}$ and $d(y)^{2} \leq 4 t$, this is proven in Lemma 2.2, so we assume $d(x)^{2} \geq$ $4 t$. However this implies $d(y)^{2} \geq t$. Otherwise we would have a contradiction:

$$
d(x, y)^{2} \geq \frac{d(x)^{2}}{2}-d(y)^{2}>2 t-t=t .
$$

So we are in the situation:

$$
d(x, y)^{2} \leq t, d(x)^{2} \geq 4 t, d(y)^{2} \geq 4 t .
$$

Applying the Harnack inequality in the cube $Q=B\left(x, 4 t^{1 / 2} / 3\right) \times(t / 4, t)$, we have

$$
G(y, t / 2 ; y, 0) \leq e^{C \sup _{Q} V t} G(x, t ; y, 0) .
$$

Since for $(z, s) \in Q, V(z) \leq \frac{a}{d(z)^{2}} \leq \frac{c}{d(x)^{2}-16 t / 9} \leq \frac{c}{t}$, we have

$$
G(y, t / 2 ; y, 0) \leq C G(x, t ; y, 0) .
$$

Applying Lemma 2.1 on $G(y, t / 2 ; y, 0)$, we have, by the doubling property,

$$
G(x, t ; y, 0) \geq c /\left|B\left(y, t^{1 / 2}\right)\right| \geq c /\left|B\left(x, t^{1 / 2}\right)\right| .
$$

This completes Part I.

Part II. When $d(x, y)^{2} \geq t$.

By Remark 1.3 we only need to show:

If $d(x, y)^{2} \geq t$, then there exist positive constants $c_{1}, c_{2}$ and $c_{3}$ such that

$$
G(x, t ; y, 0) \geq \min \left\{\left(\frac{t^{1 / 2}}{d(x)}\right)^{-c_{2}}, 1\right\} \min \left\{\left(\frac{t^{1 / 2}}{d(y)}\right)^{-c_{2}}, 1\right\} \frac{c_{1} e^{-c_{3} d(x, y)^{2} / t}}{\left|B\left(x, t^{1 / 2}\right)\right|} .
$$

Since $d(x, y)^{2} \geq t$, we have either $d(x)^{2} \geq t / 4$ or $d(y)^{2} \geq t / 4$. If both inequalities hold, then we are back to Lemma 2.3. So we can assume, by symmetry of the goal, that $d(x)^{2} \geq t / 4$ and $d(y)^{2} \leq t / 4$.

Picking a point $z$ such that $d(y, z)^{2}=t / 16$ and $d(z, 0)=d(y, 0)+d(y, z)$ and using the second entries of $G$ the same Harnack chain argument as in the proof of Lemma 2.2, we obtain, as in (2.4):

$$
G(x, t ; y, 0) \geq c\left(t^{1 / 2} / d(y)\right)^{-\alpha} G(x, t ; z, t / 2) .
$$


Since $d(x, z)^{2} \geq t / 16, d(x)^{2} \geq t / 4$ and $d(z)^{2} \geq t / 16$, we can apply Lemma 2.3 to conclude

Clearly

$$
G(x, t / 2 ; z, 0) \geq c_{1} \frac{e^{-c_{3} d(x, z)^{2} / t}}{\left|B\left(x, t^{1 / 2}\right)\right|}
$$

$$
d(x, z)^{2} \leq 2 d(x, y)^{2}+2 d(y, z)^{2} \leq 2 d(x, y)^{2}+4 d(y)^{2}+4 d(z)^{2} \leq 2 d(x, y)^{2}+2 t .
$$

Hence

$$
G(x, t / 2 ; z, 0) \geq C \frac{e^{-c_{3} d(x, y)^{2} / t}}{\left|B\left(x, t^{1 / 2}\right)\right|} .
$$

Combining (2.9) and (2.10) we have

$$
G(x, t ; y, 0) \geq C\left(\frac{t^{1 / 2}}{d(y)}\right)^{-\alpha} \frac{e^{-c_{3} d(x, y)^{2} / t}}{\left|B\left(x, t^{1 / 2}\right)\right|} .
$$

This completes the proof of Theorem 1.1 since $1 \geq \frac{t}{4 d(x)^{2}}$ by assumption.

\section{ACKNOWLEDGEMENT}

I thank Professors P. D. Milman and Yu. A. Semenov for sending me their paper [MS] before its publication and for their generosity in letting me pursue the problem. I also thank Professor G. Lieberman for useful information on the Harnack inequality. This research is supported in part by an NSF grant.

\section{REFERENCES}

[A] D.G. Aronson, Non-negative solutions of linear parabolic equations, Ann. Scuola Norm. Sup.Pisa 22 (1968), 607-694. MR 55:8553

[BG] P. Baras and J.A. Goldstein The heat equation with a singular potential, Trans. AMS, 284 (1984), 121-139. MR 85f:35099

[DS] E. B. Davies and B. Simon $L^{p}$ Norms of Non-critical Schrödinger semigroups, J. Functional Analysis 102 (1991), 95-115. MR 93e:47063

[GT] D. Gilbarg and N. S. Trudinger Elliptic partial differential equations of second order, Springer-Verlag (1983). MR 86c:35035

[LY] P. Li and S. T. Yau On the parabolic kernel of the Schrödinger operator, Acta Math 156 (1986), 153-201. MR 87f:58156

[S-C1] L. Saloff-Coste A note on Poincaré, Sobolev and Harnack inequalities, International Math. Research Notices 2 (1992), 27-38. MR 93d:58158

[S-C2] L. Saloff-Coste Uniformly elliptic operators on Riemannian manifolds, J. Diff. Geometry 36 1992, 417-450. MR 93m:58122]

[MS] Pierre D. Milman and Yu A. Semenov, De-singularizing weights and heat kernel bounds, pre-print, 1999

[Se] Yu A. Semenov, Stability of $L^{p}$-spectrum of generalized Schrödinger operators and equivalence of Green's functions, IMRN, 12 (1997), 573-593. MR 98m:47079

Department of Mathematics, University of Memphis, Memphis, Tennessee 38152 